\title{
Efficacy of transcranial direct-current stimulation (tDCS) in women with provoked vestibulodynia: study protocol for a randomized controlled trial
}

Annie Morin¹, Guillaume Léonard', Véronique Gougeon ${ }^{1}$, Guy Waddell², Yves-André Bureau², Isabelle Girard² and Mélanie Morin ${ }^{1 *}$

\begin{abstract}
Background: Provoked vestibulodynia is the most common form of vulvodynia. Despite its high prevalence and deleterious sexual, conjugal, and psychological repercussions, effective evidence-based interventions for provoked vestibulodynia remain limited. For a high proportion of women, significant pain persists despite the currently available treatments. Growing evidence suggests that the central nervous system (CNS) could play a key role in provoked vestibulodynia; thus, treatment targeting the CNS, rather than localized dysfunctions, may be beneficial for women suffering from provoked vestibulodynia. In this study, we aim to build on the promising results of a previous case report and evaluate whether transcranial direct-current stimulation, a non-invasive brain stimulation technique targeting the CNS, could be an effective treatment option for women with provoked vestibulodynia.

Methods/design: This single-center, triple-blind, parallel group, randomized, controlled trial aims to compare the efficacy of transcranial direct-current stimulation with sham transcranial direct-current stimulation in women with provoked vestibulodynia. Forty women diagnosed with provoked vestibulodynia by a gynecologist, following a standardized treatment protocol, are randomized to either active transcranial direct-current stimulation treatment for ten sessions of 20 minutes at an intensity of $2 \mathrm{~mA}$ or sham transcranial direct-current stimulation over a 2-week period. Outcome measures are collected at baseline, 2 weeks after treatment and at 3-month follow-up. The primary outcome is pain during intercourse, assessed with a numerical rating scale. Secondary measurements focus on the sexual function, vestibular pain sensitivity, psychological distress, treatment satisfaction, and the patient's global impression of change.

Discussion: To our knowledge, this study is the first randomized controlled trial to examine the efficacy of transcranial direct-current stimulation in women with provoked vestibulodynia. Findings from this trial are expected to provide significant information about a promising intervention targeting the centralization of pain in women with provoked vestibulodynia.
\end{abstract}

Trial registration: Clinicaltrials.gov, NCT02543593. Registered on September 4, 2015.

Keywords: Randomized clinical trial, Vestibulodynia, Transcranial direct-current stimulation, Chronic pain, Sexual dysfunction, Psychological distress, Treatment outcome, Dyspareunia

\footnotetext{
* Correspondence: melanie.m.morin@usherbrooke.ca

'School of Rehabilitation, Faculty of Medicine and Health Sciences, Université

de Sherbrooke, 3001 12th Avenue North, Sherbrooke, Québec, Canada

Full list of author information is available at the end of the article
} 


\section{Background}

Chronic vulvar pain represents a major health concern for women. Vulvodynia is a highly neglected chronic pain condition, affecting nearly $10 \%$ of the female population [1]. Suspected to be the foremost cause of pre-menopausal vulvodynia, provoked vestibulodynia (PVD) is characterized by an acute recurrent pain located at the vulvar vestibule (i.e., the vaginal entrance) in response to pressure application or attempted vaginal penetration [2]. It has been revealed that PVD disrupts personal lives, severely affects sexual function, and negatively impacts the quality of life [3, 4]. PVD has also been related to relationship problems and psychological distress [5]. Poorly understood and often misdiagnosed or ignored, PVD pain leads to a high personal cost for patients and substantial financial cost for society. Women with PVD often multiply their medical visits hoping to find relief and rely mainly on non-evidence-based, ineffective interventions [6].

Many empirical treatment options are proposed to women with PVD. Firstly, localized interventions such as topical lidocaine application [7], pelvic floor physical therapy [8], biofeedback technique [9], Botox ${ }^{\bullet}$ injection [10], topical use of estradiol and testosterone compounds [11], and vestibulectomy (surgery) [12] can be offered. As a second option, psychotherapeutic interventions can be proposed, which include cognitive behavioral therapy (CBT) focusing on reducing pain and improving sexual function [13]; hypnotherapy [14]; or acupuncture [15]. In addition, the use of systemic treatments, such as tricyclic antidepressants [16], anticonvulsant medication and selective serotonin reuptake inhibitors (SSRIs) [17], can also be prescribed. Despite this wide variety of treatment options, a high proportion of women with PVD are refractory to conventional treatments $[12,17,18]$, highlighting the need for novel approaches.

Recently, alterations in central pain mechanisms have been suggested to play a key role in PVD, potentially explaining the limited success obtained in some patients, with treatments targeting the pain locally in the area of the vestibule. Central sensitization has been observed in many chronic pain conditions, including chronic pelvic pain [19]. While the heightened sensitivity of peripheral pain receptors following local trauma or infection usually resolves with time, in chronic pain, this hypersensitivity is sustained and amplified by an extensive central neural network that includes the spinal dorsal horn, limbic system, and cortical structures [20]. Recent studies suggest that the pathophysiology of PVD is not only limited to the vulvar vestibule but also involves central pain processing alterations similarly to other chronic pain conditions [21]. In support of such central alterations, women with PVD have been shown to be more sensitive in areas other than the genital regions compared to controls (lower pain threshold) [21-23]. Moreover, imaging studies have revealed increased activation of brain regions associated with pain perception during painful stimuli in women with PVD [24], a pattern of results analogous to that observed in studies completed in other chronic pain populations such as fibromyalgia [25-27], irritable bowel syndrome [28-33], and idiopathic back pain [34].

Imaging studies have also shown structural changes in women suffering from PVD. Schweinhardt et al. [35] revealed higher gray matter densities in pain modulatory and stress-related areas in women with PVD, suggesting potential alterations in the supraspinal pain modulatory circuitry. These neuroanatomical changes in CNS regions related to endogenous pain modulation could explain the large-scale changes in pain sensitivity observed in women with PVD (e.g., lower pain thresholds in regions other than the vulvar vestibule). Another argument in favor of central pain mechanism alterations is the co-occurrence of other pain conditions such as orofacial pain, fibromyalgia, and irritable bowel syndrome [4, 36]. Arnold et al. [4] found that women with vulvar pain have a threefold to fourfold risk of having these concomitant pain conditions. Furthermore, similar medications (e.g., antidepressants) are commonly used to treat PVD, fibromyalgia, and irritable bowel syndrome, suggesting that these disorders may share similar pathophysiological mechanisms [37-39].

One proposed technique for modulating CNS activity in chronic pain states is noninvasive brain stimulation (NIBS). NIBS strategies aimed at modifying cortical excitability for different purposes have emerged in recent years [40]. Transcranial direct-current stimulation (tDCS) is a specific form of NIBS that has been shown effective for improving various chronic pain conditions relating to spinal cord injury [41], fibromyalgia [42], multiple sclerosis [43], painful diabetic polyneuropathy [44], pelvic pain [20, 45], and other various syndromes such as trigeminal neuralgia, poststroke pain syndrome, and back pain [46]. tDCS is a painless technique that consists of applying a low direct current through electrodes placed on the scalp to target the cerebral cortex in order to modify cortical excitability and reduce pain [40]. tDCS is a safe and simple device and could be easily integrated into a rehabilitation program.

Even if a large literature concerning tDCS in pain relief now exists [40, 47, 48], to our knowledge, only one case looking into the effect of tDCS in women with vulvodynia [49] exists. In this case report, Cecilio et al. describe the case of a woman suffering from severe chronic vulvar pain refractory to many empirical treatments (tricyclic antidepressants, anticonvulsants, and opioid analgesics) who has experienced remarkable long-lasting pain relief with tDCS. On the basis of the 
pathophysiology of chronic pain related to PVD that is similar to other chronic pain syndromes, and considering that it is a neglected women's health condition, we believe it is important to study the efficacy of this promising treatment in women with PVD. The main goal of this study is to evaluate the efficacy of active tDCS treatment in women with PVD compared to sham tDCS for pain during intercourse, as assessed with a numerical rating scale. We expect that active tDCS treatment will significantly reduce pain during intercourse (2-week posttreatment and 3-month follow-up compared to the pretreatment assessment). We expect that active tDCS treatment will be more effective for reducing pain than the sham tDCS treatment at 2-week post-treatment and 3-month follow-up.

Secondary objectives are to compare the efficacy of active $\mathrm{tDCS}$ vs. sham tDCS on sexual function, sexual satisfaction, vestibular pressure-pain threshold, psychological distress (catastrophizing, anxiety, depression, fear of pain, and vaginal penetration cognition), treatment satisfaction, and patient global impression of change (2week post-treatment and 3-month follow-up compared to pretreatment assessment).

\section{Methods/design \\ Design}

The proposed research design is a triple-blind (physiotherapist assessor, patient, and treatment provider), randomized, placebo-controlled, parallel-group trial. Participants are randomized to receive either active or sham tDCS for ten sessions of 20 minutes of stimulation over a 2-week period. As illustrated in the flow diagram (Fig. 1), the study includes three evaluation points (pretreatment assessment, post-treatment assessment performed at 2 weeks, and follow-up assessment at 3-months post-treatment). This study protocol was written in accordance with the SPIRIT statement (see Additional file 1 for the completed SPIRIT checklist).

\section{Participants}

Premenopausal women, ages $18-45$ years, suffering from pain during intercourse, are being recruited at the Research Center of the Centre Hospitalier Universitaire de Sherbrooke (CHUS). The eligibility criteria are detailed in Table 1. These criteria were selected to ensure recruitment of a homogenous sample of sexually active women with PVD and were based on previous successful studies

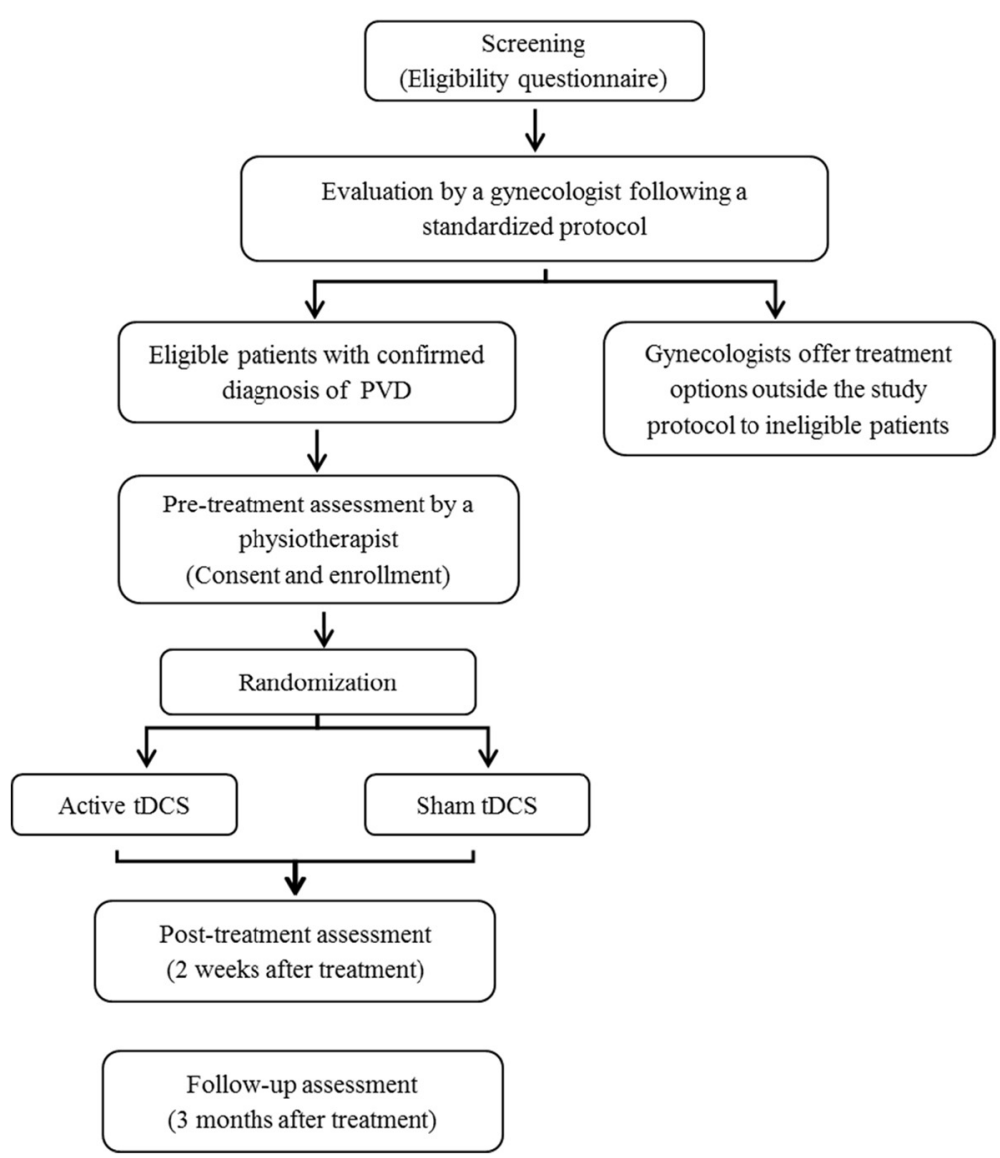

Fig. 1 Study flow diagram 
Table 1 Eligibility criteria

\begin{tabular}{|c|c|}
\hline Inclusion criteria & Exclusion criteria \\
\hline $\begin{array}{l}\text { - Experience moderate to severe pain (minimum of 5/10 } \\
\text { on a numerical rating scale (NRS)) in at least } 90 \% \text { of attempted } \\
\text { sexual intercourse } \\
\text { - Pain limited to the vestibule during vaginal intercourse and during } \\
\text { activities exerting pressure on the vestibule (tampon insertion, tight } \\
\text { jeans or pants, cycling, horseback riding) } \\
\text { - Presence of provoked vestibulodynia (PVD) for at least } 6 \text { months and } \\
\text { diagnosed according to the standardized gynecological examination } \\
\text { protocol by one of our staff gynecologists } \\
\text { - Have a stable sexual partner (sexual activity should include some } \\
\text { attempted vaginal penetrations in order to evaluate pain intensity) }\end{array}$ & $\begin{array}{l}\text { - Urogynecological conditions (e.g., active urinary tract or vaginal infection } \\
\text { or in the last } 3 \text { months, etc.) and other pelvic pathology associated } \\
\text { with pelvic pain (e.g., deep dyspareunia) } \\
\text { - Have given birth in the last year and breast feeding } \\
\text { - Anterior vulvar or vaginal surgery } \\
\text { - Refusal to refrain from other treatments } 1 \text { month prior to first treatment } \\
\text { study until the last 3-month follow-up assessment } \\
\text { - Contraindications to transcranial direct-current stimulation (tDCS) } \\
\text { (e.g., metallic implant in or near the skull, history of epilepsy, pacemaker) } \\
\text { - Previously received tDCS treatment }\end{array}$ \\
\hline
\end{tabular}

$[13,50,51]$. In order to confirm the diagnosis of PVD, the eligibility screening comprises a gynecological assessment performed by a gynecologist from our team, following a standardized protocol. The latter consists of a brief anamnesis and a comprehensive gynecological examination of the vulvar region (clitoris, small lips, interlabial furrow, and vestibule), testing for vaginal infection and sexually transmitted disease, and palpation of the uterus and appendices. This evaluation follows the diagnostic criteria defined by Friedrich [2] and more recently modified by Bergeron et al. [52], namely, (1) pain in the vestibule following touch or an attempted vaginal penetration or (2) acute pain during palpation of the vestibule region with a cotton swab. Interevaluator fidelity of this diagnosis method has been demonstrated [52] and used in several RCTs $[13,53,54]$. Therefore, vulvar pain occurring in the absence of an underlying recognizable disease and provoked spontaneously as a result of physical contact can be classified as PVD [55].

\section{Treatments}

Participants are randomized to receive ten sessions of either active or sham anodal tDCS over a period of 14 days. tDCS treatments are given once a day, during weekdays (Monday to Friday). Each session lasts 20 minutes $[20,41,44,56,57]$ and is administered by a research professional experienced in tDCS. The treatment provider is not involved in the patient assessment and is blinded to the treatment allocation by selecting a preset program of the tDCS device (NeuroConn DC stimulator). Two electrodes are applied to the subject's scalp; the anode is placed over the motor cortex (M1) [58], and the cathode, over the contralateral supraorbital area [20, 41, 44, 57, 58]. For treatment with active tDCS, the intensity of the stimulation is set at $2 \mathrm{~mA}$ for the entire duration of the treatment [20,41,42, 44, 49,56]. The parameters used in our study have been tested with many subjects in several different laboratories without side effects [59], apart from a slight itching, tingling, or burning sensation under the electrode during the first seconds of stimulation; discomfort or erythema (skin reddening) under the active electrode; and possible headache in the hours following the treatment. If the stimulation is switched on or off abruptly, the sensation of a short ocular light flash could also be felt by the participant [60]. For subjects receiving sham tDCS (placebo group), the electrodes are positioned in the same areas as the group receiving active stimulation, and the intensity is set at $2 \mathrm{~mA}$ for the first 30 seconds of treatment [57], after which the stimulation stops automatically. Just as for active tDCS, participants are advised that a brief tingling sensation may be felt at the beginning of treatment. This method is effective for preserving subject and investigator blinding [61].

In order to report the participants' adverse events during tDCS treatment, at each treatment session, the treatment provider notes the participants' side effects, and the subjects are asked to complete a logbook in which they have to report whether they experience any adverse events or pain in the vulvar region, whether related to intercourse or not. Participants also have to record their experienced vulvar pain in the same logbook between the end-of-treatment period and the 2-week post-treatment assessment.

\section{Recruitment and procedures}

Participants are recruited using different promotion strategies: (1) referrals from health professionals (gynecologist, general practitioner, sexologist, psychologist, or physiotherapist); (2) posters and leaflets distributed to women in clinics, universities, professional schools, restaurants, gyms, etc.; (3) Facebook advertisement; and 4) advertisements in local newspapers. Women interested in participating are invited to contact the research coordinator for a detailed explanation of the study and verification of their eligibility. Thereafter, participants undergo a gynecologist's examination for confirmation of their diagnoses. Once a diagnosis of PVD has been confirmed, the research assistant contacts the participants to confirm their admissibility into the study. Upon doing so, she provides the participants with instructions to follow before the assessment and, then, fixes the appointments. To avoid effects on the vestibular pain sensitivity measurements prior to each assessment, participants will be asked to refrain from smoking and consuming caffeine (coffee, tea, and energy drinks) 4 hours prior to the 
evaluation [62], to refrain from taking painkillers (Advil ${ }^{\circ}$, Motrin $^{\circ}$, Tylenol ${ }^{\circ}$, etc.) 24 hours before the evaluation [63], and to not have or attempt vaginal intercourse in the 24 hours before the evaluation [64]. After signing the informed consent, the eligible women are invited to undertake a 1-hour pretreatment assessment (baseline) with a physiotherapist specialized in pelvic-floor rehabilitation and pain assessment. During the pretreatment assessment, a structured interview is conducted to collect the patient's baseline characteristics, and standardized questionnaires are completed. Approximately $30 \mathrm{mi}-$ nutes are required to complete all questionnaires. These questionnaires have been successfully used in our previous studies, without hindering data quality [65]. A vestibular pain sensitivity assessment is also undertaken using the algometer [66]. The participants are then randomized to receive either active or sham tDCS. The same procedures are repeated at the 2-week post-treatment and 3-month followup assessments.

\section{Participant retention and protocol adherence}

As for other clinical trials, the greatest challenges pertain to participant retention. Strategies implemented to minimize attrition are as follows: (1) each participant is required to supply her contact information, such as phone number(s) and e-mail address; 2) a research assistant makes sure to remind participants of their appointments, as well as of the importance of their continuous participation; 3) all appointments are flexible to fit in with the participants' schedule (i.e., early morning, late in the evening, etc.); and 4) participants receive a $\$ 20$ compensation at three different stages of the study (the assessments) to cover travel expenses and their participation in the study. Adherence to the study protocol is also promoted throughout the trial. The physiotherapist-evaluator has received a standardized training to ensure homogeneity of procedures and reduce bias due to the evaluator. This physiotherapist-evaluator also has access to a detailed written assessment protocol Version 4 (Nov. 2, 2015). The research professional in charge of treatment is not involved in patient assessment and similarly has received proper training for the application of tDCS. The PI also carries out a weekly supervision of the physiotherapist's and professional's adherence to protocols. Moreover, the effectiveness of blinding for all physiotherapists and patients is evaluated with a questionnaire. Participants are also asked to abstain from the following PVDrelated treatments (i.e., pelvic floor physical therapy, topical lidocaine application, vestibulectomy, and psychotherapeutic and systemic interventions) during their enrollment in the study, and this is monitored at the 2-week posttreatment and 3-month follow-up assessments. In order to avoid missing data, at the end of each assessment, the physiotherapist-evaluator revises all completed questionnaires before the participant leaves.

\section{Randomization and blinding}

After the pretreatment assessment, the participants are randomized into either the active or sham tDCS treatment (ratio 1:1) using permuted blocks of two and four. The allocation is managed by an individual independent from our research team who follows a computer-generated randomization list drawn up by an independent statistician. Participants, investigators, physiotherapist assessors, and the treatment provider remain blinded to group allocation and, therefore, cannot influence the process in any way.

\section{Outcomes}

The Initiative on Methods, Measurement, and Pain Assessment in Clinical Trials (IMMPACT) has recently recommended several outcome domains for use in evaluating the effectiveness of treatment in chronic pain conditions [67]. The current protocol adheres to these recommendations, with outcomes focusing on the multiple dimensions of PVD, such as pain, sexual function, psychological distress, participant ratings of improvement and satisfaction with treatment, and the need to assess the potential impact of treatment on these different dimensions.

\section{Primary outcome measure}

As recommended by the IMMPACT $[67,68]$, participants are asked to evaluate their pain during intercourse with an NRS, 0 indicating no pain, and 10, the worst pain ever experienced. This scale has been frequently used in women with PVD [13, 69-71] and shows good reliability, validity, and responsiveness to change [13, 52, 69-71].

\section{Secondary outcome measure}

Pain measurements Different measurements of pain are recommended by the IMMPACT consensus group in order to evaluate different domains of pain $[67,72]$. Ranging from 0 to 74, the McGill-Melzack questionnaire is a 20-item scale allowing the assessment of pain quality (i.e., sensory, affective, and evaluative components). A higher total score is related to more severe pain. This world-renowned questionnaire, studied for its validity, reliability, and responsiveness to change, is commonly used in RCTs [13, 52, 73-78].

Furthermore, the third International Consultation on Sexual Medicine has emphasized the importance of assessing vestibular sensitivity [79]. Our laboratory recently developed an algometer to measure the vulvar pain threshold and tolerance in women with PVD. A gradual pressure ( 1 to 1000 grams) is applied to three distinct points of the vestibule at the 3, 6, and 9 o'clock positions [80]. Each of these pressure points is applied randomly (e.g., 3,6,9 or $3,9,6$ or $6,9,3)$. During this procedure, each participant is asked to indicate when they start to feel pain (pain threshold) and subsequently the maximal pressure they can 
tolerate (pain tolerance) (see [66] for more details). Pain intensity is assessed throughout the test using a computerized visual analog scale (CoVas). This assessment has shown good reliability and validity [80].

Sexual function measurements The Female Sexual Function Index (FSFI) is a 19-item multidimensional measure of sexual function evaluating desire, arousal, lubrication, orgasm, satisfaction, and pain. This questionnaire is frequently used in trials involving women with PVD $[13,78]$. In addition to good psychometric properties (reliability, internal consistency, and responsiveness to change) $[8,81]$, normative data are available for this questionnaire, suggesting clinical levels of dysfunctions [82]. Women considered at risk for sexual dysfunction record a FSFI total score of 26 or less [82].

In order to measure the participant's sexual satisfaction, the Global Measure of Sexual Satisfaction (GMSS) questionnaire is used. This five-item questionnaire has a total score ranging from 5 to 35 and presents good psychometric properties (a high level of internal consistency and test-retest reliability) [83]. Better sexual satisfaction will result in a higher total score. Inclusion of these questionnaires is in line with the recommendations of the IMMPACT consensus group [67] recommending evaluation of the impact of pain on functions.

Psychological distress Many studies have also determined that vulvodynia may even be a source of psychological distress [5, 84]. For these important reasons, different psychological variables are evaluated.

The Pain Catastrophizing Scale (PCS) is a 13-item questionnaire with good reliability, consistency, and responsiveness $[8,85]$ for evaluating the level of pain catastrophization, a robust predictor of pain and incapacity. The PCS yields a total score (0-52) and three subscale scores assessing rumination (0-16), magnification (0-12), and helplessness (0-24). Research at the University Centre for Research on Pain and Disability indicates that a total PCS score of 30 represents a clinically relevant level of catastrophizing [86].

The State-Trait Anxiety Inventory of Spielberger (STAI-Y) allows discrimination between anxiety as a trait of personality (T-Anxiety) or as an emotional response to a situation (S-Anxiety) [87]. This 40-item questionnaire, in which 20 items are allocated to each of the SAnxiety and T-Anxiety subscales, has previously been used in women with PVD [78] and has shown a good reliability, consistency, and responsiveness $[8,87]$. Responses for the S-anxiety scale assess intensity of current feelings "at this moment" according to the following: (1) not at all, (2) somewhat, (3) moderately so, and (4) very much so. Responses for the T-anxiety scale assess frequency of feelings "in general" according to the following: (1) almost never, (2) sometimes, (3) often, and (4) almost always [88]. Although this questionnaire was not designed or validated to be used with a cut-off score, an earlier Hungarian research used T-anxiety cut-off values as follows: less than 48 indicating no anxiety, 48 to 52 indicating mild or subclinical disorder, and higher than 52 indicating significant anxiety [89].

The Beck Depression Inventory questionnaire (BDI) [90] was recommended by Dworkin et al. [67] for assessing depression [91]. This scale has been studied for its reliability and consistency [91] and has also been used in women with PVD [78]. This 21-item questionnaire has a total score ranging from 0 to 63 . Higher total scores indicate more severe depressive symptoms. Total score cutoffs for this questionnaire are as follows: 0-9 indicating that a person is not depressed, 10-18 indicating mildmoderate depression, 19-29 indicating moderate-severe depression, and 30-63 signifying severe depression [90].

The Pain Anxiety Symptoms Scale (PASS-20), which evaluates four distinct components of pain-related anxiety (i.e., cognitive, fear, escape/avoidance, and physiological), also shows good psychometric properties [92, 93]. The total score of this 20 -item questionnaire ranges from 0 to 100. A higher total score is related to higher levels of painrelated anxiety. Individuals with acute pain presenting a total score exceeding 30 may be at an elevated risk for maladaptive pain cognitions and behaviors promoting chronic pain and disability [94].

Satisfaction with treatment and the Patient's Global Impression of Change The dimension of patient's global impression of change (PGIC) is also evaluated [95, 96]. PGIC is a validated questionnaire [97] through which patients self-report selected measures to evaluate perceived reduction in pain using a seven-point scale ranging from "very much improved" to "very much worse" and rate their treatment satisfaction over an 11-point scale that ranges from "completely satisfied" to "completely dissatisfied" in order to assess the clinical significance of the results. Subjective evaluations of pain improvement and treatment satisfaction have previously been evaluated in a randomized trial involving women with PVD [13].

\section{Ethical aspects}

The study has received ethical approval from the comité d'éthique de la recherche en santé chez l'humain du CHUS (14-169). Every potential participant is made aware that there will be no impact on her medical care if she decides not to participate in the study. Women who refuse to participate in the study or who do not fulfill the eligibility criteria are followed by the clinical staff using the most recently proposed vulvodynia guidelines. In order to minimize privacy risks, all participants are identified by an alpha-numeric code. Informed consent is obtained from 
all participants prior to trial inclusion. This trial is registered at clinicaltrials.gov NCT02543593.

\section{Statistical considerations Sample size, power, and statistical analysis methods}

A total sample size of 34 participants is sufficient to detect a clinical minimal significant difference of $2[67,98]$ on an NRS (unpaired $t$ test; $\alpha=0.05 ; \beta=0.80$, standard deviation of 2.0) based on the efficacy of tDCS in other painful conditions $[57,99]$. This estimation is conservative considering that tDCS in chronic pain demonstrated a mean pain reduction of 4.34 points [40] and that the available case study in a woman with vulvodynia showed a reduction of ten points [49]. To account for potential dropouts, a total of 40 subjects are being recruited. This estimated dropout rate $(<15 \%)$ is based on available studies and our own RCT experience in women with PVD $[13,78,100]$.

\section{Statistical analyses}

Baseline characteristics of the sample will be presented using descriptive statistics. Analyses will be done on the intention-to-treat basis. Parametric test assumptions will be examined and reported. If the data is not normally distributed, nonparametric data will be transformed. The effects of treatment on pain, sexual function and psychological distress will be examined using a mixed linear model for repeated measures. One of the factors will be the GROUP, at two levels (the treatment group receives active tDCS, and the control group, sham tDCS), while the repeated factor will be TIME (baseline, 2-week post-treatment, and 3-month follow-up assessments). Treatment efficacy will be judged on the basis of a significant GROUP*TIME interaction and contrast analysis (i.e., Tukey-Kramer contrasts when using a mixed-model approach) [101] to detect any differences. The difference between the two groups regarding satisfaction and PGIC will also be assessed using a mixed linear model for repeated measures. However, the baseline assessment will not be a repeated time-factor for these analyses. All statistical analyses will be conducted at a level of significance of 0.05 . Sensitivity analyses will be conducted to explore the effect of multiple imputation methods to replace missing data. The non-robustness of our results due to missing value will be noted and discussed.

\section{Discussion}

This is the first study to evaluate the efficacy of tDCS for reducing pain during intercourse in women with PVD. Considering that the current literature on PVD pathophysiology has converged toward the cause being central pain sensitization, tDCS is a promising treatment targeting central mechanisms rather than focusing strictly on the vestibule.
The plausibility of treatment effects relies on a large body of evidence supporting treatment efficacy in various chronic pain conditions [40], including a case study showing complete pain resolution in a woman with vulvodynia [49]. The underlying mechanisms of action also support the potential short and long-lasting effects in women with PVD. Immediate effects of tDCS are explained by polarity-dependent shifts of the resting membrane potential and consequent alteration of corticospinal excitability at the stimulation site. Alteration leads to facilitation or inhibition of the superficial structures and of deeper and more remote brain areas related to pain modulation, such as the periaqueducal gray, insula, and thalamus [40]. Thus, the immediate effects of tDCS are a consequence of neuronal hyperexcitability caused by the anode or hypoexcitability induced by the cathode [47], whereas the long-lasting effects of tDCS seem to depend on N-methyl-D-aspartate (NMDA) receptorefficacy changes [102]. In patients with chronic pain, the anode is commonly placed over the motor cortex (M1) [103]. According to functional imaging studies, stimulation of the motor cortex modulates activity in the limbic system, brainstem and spinal cord, which are all involved in the emotional component of pain [104-106]. Therefore, even if the genital zone is located deeper in the central sulcus of the somato-sensorial cortex, available evidence suggests that the mechanism of action of $\mathrm{tDCS}$ for reducing pain involves the emotional component of pain.

This randomized placebo-controlled trial addresses the urgent need to provide evidence-based treatments for women with PVD. In addition, blinding of participants, evaluators, and investigators, as well as the use of recommended validated tools, strengthened our study design. Given the efficacy found in various chronic pain conditions and the promising results obtained in a case report study in a woman with vulvodynia, this new treatment avenue may give hope to women who have experienced failure with the available localized treatments.

\section{Trial status}

The recruitment of participants is ongoing at the Research Center of the CHUS. Forty women with PVD are planned to be recruited and randomized from December 2014 to May 2016.

\section{Additional file}

Additional file 1: SPIRIT checklist. Completed SPIRIT checklist. (PDF 51 kb)

Abbreviations

ANOVA: analysis of variance; BDI: Beck depression inventory questionnaire; CBT: cognitive behavioral therapy; CHUS: Centre Hospitalier Universitaire de Sherbrooke; CNS: central nervous system; CoVas: computerized visual analog scale; FSFI: female sexual function index; GMSS: global measure of sexual satisfaction; IASTA: state-trait anxiety inventory of Spielberger; 
IMMPACT: Initiative on Methods, Measurement, and Pain Assessment in Clinical Trials; M1: motor cortex; mA: milliamperes; MPQ: McGill pain questionnaire; NIBS: non-invasive brain stimulation; NMDA: N-methyl-D-aspartate; NRS: numeric rating scale; PASS-20: pain anxiety symptoms scale; PCS: pain catastrophizing scale; PGIC: patient's global impression of change; PVD: provoked vestibulodynia; RCT: randomized controlled trial; SSRIs: selective serotonin reuptake inhibitors; tDCS: transcranial direct-current stimulation.

\section{Competing interests}

The authors declare that they have no competing interests.

\section{Authors' contributions}

$A M, G L$, and MM conceived the study and helped draft the manuscript. VG, $\mathrm{GW}, \mathrm{YAB}$, and IG provided assistance in study design and implementation. All authors read and approved the final manuscript.

\section{Acknowledgments}

This research is funded by an operating grant from the Research Center of the CHUS. Drs. Morin and Léonard are supported by a Junior 1 Research Scholar of the Fonds de la Recherche du Québec - Santé (FRQ-S).

\section{Author details}

${ }^{1}$ School of Rehabilitation, Faculty of Medicine and Health Sciences, Université de Sherbrooke, 3001 12th Avenue North, Sherbrooke, Québec, Canada. 2Department of Obstetrics Gynecology, Faculty of Medicine and Health Sciences, Université de Sherbrooke, 3001 12th Avenue North, Sherbrooke, Québec, Canada.

\section{Received: 15 January 2016 Accepted: 29 April 2016} Published online: 14 May 2016

\section{References}

1. Harlow BL, Kunitz CG, Nguyen RHN, Rydell SA, Turner RM, Maclehose RF. Prevalence of symptoms consistent with a diagnosis of vulvodynia: population-based estimates from 2 geographic regions. Am J Obstet Gynecol. 2014;210(1):40.e1-.e8. doi:10.1016/j.ajog.2013.09.033.

2. Friedrich Jr EG. Vulvar vestibulitis syndrome. J Reprod Med. 1987;32(2):110-4.

3. Payne KA, Binik YM, Amsel R, Khalife S. When sex hurts, anxiety and fear orient attention towards pain. Eur J Pain (London, England). 2005:9(4):427-36. doi:10.1016/j.ejpain.2004.10.003.

4. Arnold LD, Bachmann GA, Rosen R, Kelly S, Rhoads GG. Vulvodynia: characteristics and associations with comorbidities and quality of life Obstet Gynecol. 2006;107(3):617-24. doi:10.1097/01.AOG.0000199951. 26822.27.

5. Desrochers $G$, Bergeron S, Landry $T$, Jodoin M. Do psychosexual factors play a role in the etiology of provoked vestibulodynia? a critical review. J Sex Marital Ther. 2008:34(3):198-226. doi:10.1080/00926230701866083.

6. Gordon AS, Panahian-Jand M, McComb F, Melegari C, Sharp S. Characteristics of women with vulvar pain disorders: responses to a web-based survey. J Sex Marital Ther. 2003;29 Suppl 1:45-58. doi:10.1080/713847126.

7. Haefner HK, Collins ME, Davis GD, Edwards L, Foster DC, Hartmann ED, et al. The vulvodynia guideline. J Low Genit Tract Dis. 2005;9(1):40-51.

8. Goldfinger C, Pukall CF, Gentilcore-Saulnier E, McLean L, Chamberlain S. A prospective study of pelvic floor physical therapy: pain and psychosexual outcomes in provoked vestibulodynia. J Sex Med. 2009;6(7):1955-68. doi:10. 1111/j.1743-6109.2009.01304.x.

9. Danielsson I, Torstensson T, Brodda-Jansen G, Bohm-Starke N. EMG biofeedback versus topical lidocaine gel: a randomized study for the treatment of women with vulvar vestibulitis. Acta Obstet Gynecol Scand. 2006;85(11):1360-7. doi:10.1080/00016340600883401.

10. Dykstra DD, Presthus J. Botulinum toxin type A for the treatment of provoked vestibulodynia: an open-label, pilot study. J Reprod Med. 2006; 51(6):467-70.

11. Goldstein A, Burrows L, Goldstein I. Can oral contraceptives cause vestibulodynia? J Sex Med. 2010;7(4 Pt 1):1585-7. doi:10.1111/j.1743-6109. 2009.01685.x.

12. Landry T, Bergeron S, Dupuis MJ, Desrochers G. The treatment of provoked vestibulodynia: a critical review. Clin J Pain. 2008;24(2):155-71. doi:10.1097/ AJP.0b013e31815aac4d.
13. Bergeron S, Binik YM, Khalife S, Pagidas K, Glazer HI, Meana M, et al. A randomized comparison of group cognitive-behavioral therapy, surface electromyographic biofeedback, and vestibulectomy in the treatment of dyspareunia resulting from vulvar vestibulitis. Pain. 2001;91(3):297-306.

14. Pukall C, Kandyba K, Amsel R, Khalife S, Binik Y. Effectiveness of hypnosis for the treatment of vulvar vestibulitis syndrome: a preliminary investigation. J Sex Med. 2007;4(2):417-25. doi:10.1111/j.1743-6109.2006.00425.x.

15. Powell J, Wojnarowska F. Acupuncture for vulvodynia. J R Soc Med. 1999; 92(11):579-81.

16. Reed BD, Caron AM, Gorenflo DW, Haefner HK. Treatment of vulvodynia with tricyclic antidepressants: efficacy and associated factors. J Low Genit Tract Dis. 2006;10(4):245-51. doi:10.1097/01.Igt.0000225899.75207.0a.

17. Pukall CF, Smith KB, Chamberlain SM. Provoked vestibulodynia. Womens Health (Lond Engl). 2007;3(5):583-92. doi:10.2217/17455057.3.5.583.

18. Stockdale CK, Lawson HW. 2013 Vulvodynia guideline update. J Low Genit Tract Dis. 2014;18(2):93-100. doi:10.1097/LGT.0000000000000021.

19. Woolf CJ. Central sensitization: uncovering the relation between pain and plasticity. Anesthesiology. 2007;106(4):864-7. doi:10.1097/01.anes. 0000264769.87038 .55

20. Simis M, Reidler JS, Duarte Macea D, Moreno Duarte I, Wang X, Lenkinski R, et al. Investigation of central nervous system dysfunction in chronic pelvic pain using magnetic resonance spectroscopy and noninvasive brain stimulation. Pain Pract. 2015;15(5):423-32. doi:10.1111/papr.12202.

21. Giesecke J, Reed BD, Haefner HK, Giesecke T, Clauw DJ, Gracely RH. Quantitative sensory testing in vulvodynia patients and increased peripheral pressure pain sensitivity. Obstet Gynecol. 2004;104(1):126-33. doi:10.1097/01. AOG.0000129238.49397.4e

22. Granot M, Friedman M, Yarnitsky D, Zimmer EZ. Enhancement of the perception of systemic pain in women with vulvar vestibulitis. BJOG. 2002;109(8):863-6.

23. Pukall CF, Binik YM, Khalife $S$, Amsel R, Abbott FV. Vestibular tactile and pain thresholds in women with vulvar vestibulitis syndrome. Pain. 2002;96(1-2):163-75

24. Pukall CF, Strigo IA, Binik YM, Amsel R, Khalife S, Bushnell MC. Neural correlates of painful genital touch in women with vulvar vestibulitis syndrome. Pain. 2005:115(1-2):118-27. doi:10.1016/j.pain.2005.02.020

25. Cook DB, Lange G, Ciccone DS, Liu WC, Steffener J, Natelson BH. Functional imaging of pain in patients with primary fibromyalgia. J Rheumatol. 2004;31(2):364-78.

26. Gracely RH, Petzke F, Wolf JM, Clauw DJ. Functional magnetic resonance imaging evidence of augmented pain processing in fibromyalgia. Arthritis Rheum. 2002:46(5):1333-43. doi:10.1002/art.10225.

27. Gracely RH, Geisser ME, Giesecke T, Grant MA, Petzke F, Williams DA, et al. Pain catastrophizing and neural responses to pain among persons with fibromyalgia. Brain. 2004;127(Pt 4):835-43. doi:10.1093/brain/awh098.

28. Bernstein CN, Frankenstein UN, Rawsthorne P, Pitz M, Summers R, Mclntyre MC. Cortical mapping of visceral pain in patients with $\mathrm{Gl}$ disorders using functional magnetic resonance imaging. Am J Gastroenterol. 2002;97(2): 319-27. doi:10.1111/j.1572-0241.2002.05464.x.

29. Chang L, Berman S, Mayer EA, Suyenobu B, Derbyshire S, Naliboff B, et al. Brain responses to visceral and somatic stimuli in patients with irritable bowel syndrome with and without fibromyalgia. Am J Gastroenterol. 2003; 98(6):1354-61. doi:10.1111/j.1572-0241.2003.07478.x.

30. Mertz H, Morgan V, Tanner G, Pickens D, Price R, Shyr Y, et al. Regional cerebral activation in irritable bowel syndrome and control subjects with painful and nonpainful rectal distention. Gastroenterology. 2000:118(5):842-8.

31. Naliboff BD, Derbyshire SW, Munakata J, Berman S, Mandelkern M, Chang L, et al. Cerebral activation in patients with irritable bowel syndrome and control subjects during rectosigmoid stimulation. Psychosom Med. 2001;63(3):365-75.

32. Ringel Y, Drossman DA, Turkington TG, Bradshaw B, Hawk TC, Bangdiwala S, et al. Regional brain activation in response to rectal distension in patients with irritable bowel syndrome and the effect of a history of abuse. Dig Dis Sci. 2003:48(9):1774-81.

33. Verne GN, Himes NC, Robinson ME, Gopinath KS, Briggs RW, Crosson B, et al. Central representation of visceral and cutaneous hypersensitivity in the irritable bowel syndrome. Pain. 2003;103(1-2):99-110.

34. Giesecke T, Gracely RH, Grant MA, Nachemson A, Petzke F, Williams DA, et al. Evidence of augmented central pain processing in idiopathic chronic low back pain. Arthritis Rheum. 2004;50(2):613-23. doi:10.1002/art.20063.

35. Schweinhardt P, Kuchinad A, Pukall CF, Bushnell MC. Increased gray matter density in young women with chronic vulvar pain. Pain. 2008:140(3):411-9. doi:10.1016/j.pain.2008.09.014. 
36. Zolnoun DA, Rohl J, Moore CG, Perinetti-Liebert C, Lamvu GM, Maixner W. Overlap between orofacial pain and vulvar vestibulitis syndrome. Clin J Pain. 2008;24(3):187-91. doi:10.1097/AJP.0b013e318159f976.

37. Hauser W, Petzke F, Uceyler N, Sommer C. Comparative efficacy and acceptability of amitriptyline, duloxetine and milnacipran in fibromyalgia syndrome: a systematic review with meta-analysis. Rheumatology (Oxford) 2011;50(3):532-43. doi:10.1093/rheumatology/keq354

38. Chao GQ, Zhang S. A meta-analysis of the therapeutic effects of amitriptyline for treating irritable bowel syndrome. Intern Med (Tokyo, Japan). 2013;52(4):419-24.

39. Brown CS, Wan J, Bachmann G, Rosen R. Self-management, amitriptyline, and amitripyline plus triamcinolone in the management of vulvodynia. $J$ Womens Health (Larchmt). 2009;18(2):163-9. doi:10.1089/jwh.2007.0676.

40. Vaseghi B, Zoghi M, Jaberzadeh S. Does anodal transcranial direct current stimulation modulate sensory perception and pain? a meta-analysis study. Clin Neurophysiol. 2014;125(9):1847-58. doi:10.1016/j.clinph.2014.01.020.

41. Fregni F, Boggio PS, Lima MC, Ferreira MJ, Wagner T, Rigonatti SP, et al. A sham-controlled, phase II trial of transcranial direct current stimulation for the treatment of central pain in traumatic spinal cord injury. Pain. 2006; 122(1-2):197-209. doi:10.1016/j.pain.2006.02.023.

42. Fregni F, Gimenes R, Valle AC, Ferreira MJ, Rocha RR, Natalle L, et al. A randomized, sham-controlled, proof of principle study of transcranial direct current stimulation for the treatment of pain in fibromyalgia. Arthritis Rheum. 2006;54(12):3988-98. doi:10.1002/art.22195.

43. Mori F, Codeca C, Kusayanagi H, Monteleone F, Buttari F, Fiore S, et al. Effects of anodal transcranial direct current stimulation on chronic neuropathic pain in patients with multiple sclerosis. J Pain. 2010;11(5):436-42. doi:10.1016/j.jpain.2009.08.011.

44. Kim YJ, Ku J, Kim HJ, Im DJ, Lee HS, Han KA, et al. Randomized, sham controlled trial of transcranial direct current stimulation for painful diabetic polyneuropathy. Ann Rehabil Med. 2013;37(6):766-76. doi:10.5535/arm.2013.37.6.766.

45. Fenton BW, Palmieri PA, Boggio P, Fanning J, Fregni F. A preliminary study of transcranial direct current stimulation for the treatment of refractory chronic pelvic pain. Brain Stimul. 2009;2(2):103-7. doi:10.1016/j.brs.2008.09.009.

46. Antal A, Terney D, Kuhnl S, Paulus W. Anodal transcranial direct current stimulation of the motor cortex ameliorates chronic pain and reduces short intracortical inhibition. J Pain Symptom Manage. 2010;39(5):890-903. doi:10. 1016/j.jpainsymman.2009.09.023.

47. Thibaut A, Chatelle C, Gosseries O, Laureys S, Bruno MA. Transcranial direct current stimulation: a new tool for neurostimulation. Rev Neurol. 2013; 169(2):108-20. doi:10.1016/j.neurol.2012.05.008.

48. O'Connell NE, Wand BM, Marston L, Spencer S, Desouza LH. Non-invasive brain stimulation techniques for chronic pain: a report of a Cochrane systematic review and meta-analysis. Eur J Phys Rehabil Med. 2011;47(2):309-26.

49. Cecilio SB, Zaghi S, Cecilio LB, Correa CF, Fregni F. Exploring a novel therapeutic approach with noninvasive cortical stimulation for vulvodynia. Am J Obstet Gynecol. 2008;199(6):e6-7. doi:10.1016/j.ajog.2008.08.034.

50. Morin M, Bergeron S, Khalife S, Mayrand MH, Binik YM. Morphometry of the pelvic floor muscles in women with and without provoked vestibulodynia using 4D ultrasound. J Sex Med. 2014;11(3):776-85. doi:10.1111/jsm.12367.

51. Bergeron S, Brown C, Lord MJ, Oala M, Binik YM, Khalife S. Physical therapy for vulvar vestibulitis syndrome: a retrospective study. J Sex Marital Ther. 2002;28(3):183-92

52. Bergeron S, Binik YM, Khalife S, Pagidas K, Glazer HI. Vulvar vestibulitis syndrome: reliability of diagnosis and evaluation of current diagnostic criteria. Obstet Gynecol. 2001;98(1):45-51.

53. Corsini-Munt S, Bergeron S, Rosen NO, Steben M, Mayrand MH, Delisle I, et al. A comparison of cognitive-behavioral couple therapy and lidocaine in the treatment of provoked vestibulodynia: study protocol for a randomized clinical trial. Trials. 2014;15:506. doi:10.1186/1745-6215-15-506.

54. Morin M, Dumoulin C, Bergeron S, Mayrand MH, Khalife S, Waddell G, et al. Randomized clinical trial of physiotherapy treatment compared to overnight lidocaine ointment in women suffering from provoked vestibulodynia: design and methods. Contemp Clinic Trials. 2016:46:52-9. doi:10.1016/j.cct.2015.11.013.

55. Moyal-Barracco M, Lynch PJ. 2003 ISSVD terminology and classification of vulvodynia: a historical perspective. J Reprod Med. 2004;49(10):772-7.

56. Reidler JS, Mendonca ME, Santana MB, Wang X, Lenkinski R, Motta AF, et al. Effects of motor cortex modulation and descending inhibitory systems on pain thresholds in healthy subjects. J Pain. 2012;13(5):450-8. doi:10.1016/j.jpain.2012.01.005.
57. Soler MD, Kumru H, Pelayo R, Vidal J, Tormos JM, Fregni F, et al. Effectiveness of transcranial direct current stimulation and visual illusion on neuropathic pain in spinal cord injury. Brain. 2010;133(9):2565-77. doi:10. 1093/brain/awq184.

58. Nitsche MA, Paulus W. Sustained excitability elevations induced by transcranial DC motor cortex stimulation in humans. Neurology. 2001:57(10):1899-901.

59. Fregni F, Nitsche MA, Loo CK, Brunoni AR, Marangolo P, Leite J, et al. Regulatory considerations for the clinical and research use of transcranial direct current stimulation (tDCS): review and recommendations from an expert panel. Clin Res Regul Aff. 2014;32(1):22-35. doi:10.3109/10601333.2015.980944.

60. Nitsche MA, Liebetanz D, Lang N, Antal A, Tergau F, Paulus W. Safety criteria for transcranial direct current stimulation (tDCS) in humans. Clin Neurophysiol. 2003;114(11):2220-2. author reply 2-3.

61. Gandiga PC, Hummel FC, Cohen LG. Transcranial DC stimulation (tDCS): a tool for double-blind sham-controlled clinical studies in brain stimulation Clin Neurophysiol. 2006;117(4):845-50. doi:10.1016/j.clinph.2005.12.003.

62. Marchand S, Li J, Charest J. Effects of caffeine on analgesia from transcutaneous electrical nerve stimulation. N Engl J Med. 1995;333(5):325-6. doi:10.1056/NEJM199508033330521.

63. Leonard G, Goffaux P, Mathieu D, Blanchard J, Kenny B, Marchand S. Evidence of descending inhibition deficits in atypical but not classical trigeminal neuralgia. Pain. 2009;147(1-3):217-23. doi:10.1016/j.pain.2009.09.009.

64. Bohm-Starke N, Hilliges M, Blomgren B, Falconer C, Rylander E. Increased blood flow and erythema in the posterior vestibular mucosa in vulvar vestibulitis(1). Obstet Gynecol. 2001;98(6):1067-74.

65. Morin M, Dumoulin C, Bergeron S, Mayrand MH, Khalife S, Waddell G et al., editors. PVD Study Group. Randomized controlled trial of multimodal physiotherapy treatment compared to overnight topical lidocaine in women suffering from provoked vestibulodynia. 40th Annual Meeting of the International Urogynecological Association; 2015; Nice, France, Int Urogynecol J, 1(Supp - June 2015), PP22.

66. Cyr M-P, Bourbonnais D, Pinard A, Dubois O, Morin M. Reliability and convergent validity of the algometer for vestibular pain assessment in women with provoked vestibulodynia. Pain Med. 2015. doi:10.1093/pm/pnv069.

67. Dworkin RH, Turk DC, Farrar JT, Haythornthwaite JA, Jensen MP, Katz NP, et al. Core outcome measures for chronic pain clinical trials: IMMPACT recommendations. Pain. 2005;113(1-2):9-19. doi:10.1016/j.pain.2004.09.012.

68. Katz J, Melzack R. Measurement of pain. Surg Clin North Am. 1999;79(2):231-52.

69. Morin M, Bergeron S, Khalife S, Binik I, Ouellet S. Dynamometric assessment of the pelvic floor muscle function in women with and without provoked vestibulodynia. NeurourolUrodyn. 2010;29:1140-1.

70. Morin M, Bergeron S, Khalife S, Binik I, Mayrand MH. Morphometry of the pelvic floor muscles in women with and without provoked vestibuylodynia using 3D/4D ultrasonography. J Sex Med. 2011;8 suppl 2:56.

71. Bergeron S, Khalife S, Dupuis MJ, editors. Provoked vestibulodynia: a randomized comparison of cognitive-behavioral therapy and medical management. Communicatoin presentée dans le cadre d'un symposium au congrès annuel de l'International Society for the Study of Women and Sexual Health; 2008.

72. Turk DC, Dworkin RH, Burke LB, Gershon R, Rothman M, Scott J, et al. Developing patient-reported outcome measures for pain clinical trials: IMMPACT recommendations. Pain. 2006;125(3):208-15. doi:10.1016/j.pain.2006.09.028.

73. Melzack R. The McGill pain questionnaire: major properties and scoring methods. Pain. 1975;1(3):277-99.

74. Davidoff G, Guarracini M, Roth E, Sliwa J, Yarkony G. Trazodone hydrochloride in the treatment of dysesthetic pain in traumatic myelopathy: a randomized, double-blind, placebo-controlled study. Pain. 1987;29(2):151-61.

75. Hays H, Hagen N, Thirlwell M, Dhaliwal H, Babul N, Harsanyi Z, et al. Comparative clinical efficacy and safety of immediate release and controlled release hydromorphone for chronic severe cancer pain. Cancer. 1994;74(6):1808-16.

76. Bansal D, Bhansali A, Hota D, Chakrabarti A, Dutta P. Amitriptyline vs. pregabalin in painful diabetic neuropathy: a randomized double blind clinical trial. Diabet Med. 2009;26(10):1019-26. doi:10.1111/j.1464-5491.2009.02806.x.

77. Moy I, Milad MP, Barnes R, Confino E, Kazer RR, Zhang X. Randomized controlled trial: effects of acupuncture on pregnancy rates in women undergoing in vitro fertilization. Fertil Steril. 2011;95(2):583-7. doi:10.1016/j. fertnstert.2010.05.024.

78. Foster DC, Kotok MB, Huang LS, Watts A, Oakes D, Howard FM, et al. Oral desipramine and topical lidocaine for vulvodynia: a randomized controlled trial. Obstet Gynecol. 2010:116(3):583-93 doi:10.1097/AOG. ob013e3181e9e0ab. 
79. van Lankveld JJ, Granot M, Weijmar Schultz WC, Binik YM, Wesselmann U, Pukall CF, et al. Women's sexual pain disorders. J Sex Med. 2010;7(1 Pt 2): 615-31. doi:10.1111/j.1743-6109.2009.01631.x.

80. Cyr MP, Morin M, Pinard A, Dubois O, Bourbonnais D. Reliability and validity of a new instrument for vestibular pain assessment in women with provoked vestibulodynia. J Sex Med. 2014;11 suppl 4:213-37.

81. Rosen R, Brown C, Heiman J, Leiblum S, Meston C, Shabsigh R, et al. The female sexual function index (FSFI): a multidimensional self-report instrument for the assessment of female sexual function. J Sex Marital Ther. 2000:26(2):191-208. doi:10.1080/009262300278597.

82. Wiegel M, Meston C, Rosen R. The female sexual function index (FSFI): cross-validation and development of clinical cutoff scores. J Sex Marital Ther. 2005:31(1):1-20. doi:10.1080/00926230590475206.

83. Lawrance K, Byers ES. Sexual satisfaction in long-term heterosexual relationship: The Interpersonal exchange model of sexual satisfaction. Pers Relat. 1995;2:267-85.

84. Meana M, Binik YM, Khalife S, Cohen DR. Biopsychosocial profile of women with dyspareunia. Obstet Gynecol. 1997;90(4 Pt 1):583-9.

85. French D, Noel M, Vigneau F, French J, Cyr C, Evans R. PCS-CF: A Frenchlanguage, French-Canadian adaptation of the pain catastrophizing scale. Can J Behav Sci. 2005;37(3):181-92.

86. Sullivan MJL, Bishop SR, Pivik J. The pain catastrophizing scale: development and validation. Psychol Assess. 1995:7(4):524-32.

87. Gauthier J, Bouchard S. Adaptation canadienne-française de la forme révisée du state-trait anxiety inventory de Spielberger. [A French-Canadian adaptation of the revised version of Spielberger's state-trait anxiety inventory.]. Can J Behav Sci. 1993;25(4):559-78. doi:10.1037/h0078881.

88. Julian ப. Measures of anxiety. Arthritis Care Res. 2011;63(0 11):10.1002/acr.20561. doi:10.1002/acr.20561.

89. Stauder A, Kovacs M. Anxiety symptoms in allergic patients: identification and risk factors. Psychosom Med. 2003;65(5):816-23.

90. Beck AT, Steer RA, Brown GK. Manual for the Beck depression inventory-II. San Antonio, TX: Psychological Corp; 1996.

91. Bourque P, Beaudette D. Étude psychometrique du questionnaire de dépression de Beck auprès d'un échantillon d'étudiants universitaires francophones. [Psychometric study of the Beck Depression Inventory on a sample of French-speaking university students.]. Can J Behav Sci. 1982;14(3):211-8. doi:10.1037/h0081254

92. Coons MJ, Hadjistavropoulos HD, Asmundson GJ. Factor structure and psychometric properties of the pain anxiety symptoms scale-20 in a community physiotherapy clinic sample. Eur J Pain (London, England). 2004; 8(6):511-6. doi:10.1016/j.ejpain.2003.11.018.

93. McCracken LM, Dhingra L. A short version of the pain anxiety symptoms scale (PASS-20): preliminary development and validity. Pain Res Manag. 2002;7(1):45-50.

94. Abrams MP, Carleton RN, Asmundson GJ. An exploration of the psychometric properties of the PASS-20 with a nonclinical sample. J Pain. 2007;8(11):879-86. doi:10.1016/j.jpain.2007.06.004

95. Farrar JT, Young Jr JP, LaMoreaux L, Werth JL, Poole RM. Clinical importance of changes in chronic pain intensity measured on an 11-point numerical pain rating scale. Pain. 2001;94(2):149-58.

96. Hurst $\mathrm{H}$, Bolton J. Assessing the clinical significance of change scores recorded on subjective outcome measures. J Manipulative Physiol Ther. 2004;27(1):26-35. doi:10.1016/j.jmpt.2003.11.003.

97. Farrar JT, Berlin JA, Strom BL. Clinically important changes in acute pain outcome measures: a validation study. J Pain Symptom Manage. 2003;25(5):406-11.

98. Farrar JT, Portenoy RK, Berlin JA, Kinman JL, Strom BL. Defining the clinically important difference in pain outcome measures. Pain. 2000;88(3):287-94.

99. Borckardt JJ, Romagnuolo J, Reeves ST, Madan A, Frohman H, Beam W, et al. Feasibility, safety, and effectiveness of transcranial direct current stimulation for decreasing post-ERCP pain: a randomized, sham-controlled, pilot study. Gastrointest Endosc. 2011;73(6):1158-64. doi:10.1016/j.gie.2011.01.050.

100. Morin M, Dumoulin C, Bergeron S, Mayrand MH, Khalife S, Waddell G, et al. Randomized clinical trial of multimodal physiotherapy treatment compared to overnight lidocaine ointment in women with provoked vestibulodynia: design and methods. Contemp Clin Trials. 2016;46:52-9. doi:10.1016/j.cct.2015.11.013.

101. Neter J, Wasserman W, Kutner MH. Applied linear statistical models. Boston: Irwin; 1990.

102. Liebetanz D, Nitsche MA, Tergau F, Paulus W. Pharmacological approach to the mechanisms of transcranial DC-stimulation-induced after-effects of human motor cortex excitability. Brain. 2002;125(Pt 10):2238-47.
103. Valeriani M, Restuccia D, Di Lazzaro V, Oliviero A, Profice P, Le Pera D, et al. Inhibition of the human primary motor area by painful heat stimulation of the skin. Clin Neurophysiol. 1999;110(8):1475-80.

104. Peyron R, Faillenot I, Mertens P, Laurent B, Garcia-Larrea L. Motor cortex stimulation in neuropathic pain: correlations between analgesic effect and hemodynamic changes in the brain: a PET study. Neuroimage. 2007;34(1): 310-21. doi:10.1016/j.neuroimage.2006.08.037.

105. Garcia-Larrea L, Peyron R, Mertens P, Gregoire MC, Lavenne F, Le Bars D, et al. Electrical stimulation of motor cortex for pain control: a combined PETscan and electrophysiological study. Pain. 1999;83(2):259-73.

106. Garcia-Larrea L, Peyron R. Motor cortex stimulation for neuropathic pain: from phenomenology to mechanisms. Neuroimage. 2007;37 Suppl 1:S71-9. doi:10.1016/j.neuroimage.2007.05.062

\section{Submit your next manuscript to BioMed Central and we will help you at every step:}

- We accept pre-submission inquiries

- Our selector tool helps you to find the most relevant journal

- We provide round the clock customer support

- Convenient online submission

- Thorough peer review

- Inclusion in PubMed and all major indexing services

- Maximum visibility for your research

Submit your manuscript at www.biomedcentral.com/submit
) Biomed Central 